\title{
The impact of Nordic walking trainings on social well-being of women at the age of $\mathbf{5 0 - 6 0}$
}

\author{
Antonina A. Soboleva ${ }^{1}$, Galiya S. Iskakova ${ }^{1}$, Rezeda R. Khasanova ${ }^{1, a}$, Vladimir I. Andreev ${ }^{1}$,Oleg I. Zagrevsky ${ }^{2}$, Darya U. \\ Nikonova ${ }^{1}$ \\ ${ }^{1}$ Tomsk Polytechnic University, 634050 Lenin Avenue, 30, Tomsk, Russia \\ ${ }^{2}$ Tomsk State University, 634050 Lenin Avenue, 36, Tomsk, Russia
}

\begin{abstract}
Prevention of falls aims to increase the strength of large groups of muscles, mainly in the lower limbs, as well as to improve gait, balance, and coordination parameters. Nordic walking (NW) is a new type of walking training; the main purpose of NW is to involve the muscles that are not used during normal walking, which enables the performance of high intensity exercises at a relatively low level of perceived exertion. The article deals with the relevance of the problem of raising the health level and working capacity of mature and elderly-aged population. The importance of regular physical exercises as a universal means of satisfying the human body in motion has proved to be substantial. The research results in a positive impact of NW training on the functional status and health of women under the age of $50-60$, the optimal structure of correctly graduated exercises during Nordic walking trainings in winter and snow-free periods. The quality of women's life is determined and improved. NW is a form of physical activity which is recommended along with physiotherapy for the elderly to prevent musculoskeletal disorders, vascular diseases, cardiovascular diseases, thus improving people's physical, and, hence, social welfare.
\end{abstract}

\section{Introduction}

It is known that aging originates in cell structures and their changes arise due to violation of various body functions. Physical exercises help people to get rid of stress. By nature, a person is created in such a way that, in case of danger, adrenaline rushes into the blood allowing people to physically sharpen and accelerate their reactions. In ancient times, people were forced to fight for their existence, so these reactions were necessary for survival. In the modern world, we are constantly under stress, but as our life in this case is not directly threatened, it does not seem necessary to act physically in self-defense, and our body behaves typically. Everyone can undoubtedly feel how his/her body reacts to an unexpected stimulus. This happens in a state of tension, which occurs, for example, during examinations (the heart starts to beat faster, breathing quickens, hands sweat, there is a sensation of dry mouth, etc.) [1]. In this state the human body is ready for the activity, as it is under stress, but the actual physical activity rarely follows. Uncertainty about the future, continuous haste and wrong, monotonous work position causes a constant state of tension in muscles. This tension prevents the normal physical and mental activity, which, in its turn, has a detrimental effect on the heart and respiratory organs. A physical activity is an ideal tool to relieve from everyday stress in order to achieve relaxation and well-being sensation. [2, 3]

One of the most effective means of positive and beneficial influence on the condition of the body is exercises. The movements, which are based on muscle contractions, are an essential life necessity for a human being. Universal means of full satisfaction of the body in motion is regular physical exercises. [4, 5] They have a versatile favourable impact on the body, stimulate the work of all physiological systems, contribute to the increase of working capacity, prevent from premature aging and are available to almost everyone.

Performing a low-intensity physical activity (rowing, walking, cycling, jogging, skiing, Nordic walking) increases energy losses and contributes to gradual weight reduction in case of overweight people. In addition, it hinders its formation when the weight is normal. It is known that obesity contributes to the development of hypertension, cancer, atherosclerosis, diabetes, accelerates the process of aging. In addition, moderate physical activity temporarily increases blood sugar levels, reduces appetite and eliminates the risk of binge eating. [2]

\footnotetext{
a Corresponding author: hasanova_rezeda@mail.ru
} 
Oxidation processes occur in every cell, but during exercises, they are intensive in the tissues of the musculoskeletal system, especially in the muscles. For this reason, people who are regularly engaged in physical activity, have hypertrophied muscles, which significantly increases strength and speed of muscles reductions [1]

Due to motor activity in a human's body, calcium and other minerals are better absorbed, and therefore they strengthened bone tissue. People, who lead a sedentary lifestyle, especially after the age of fifty, have a progressive decrease of calcium in the body and there is an increase in fragility of the bone tissue $[2,6]$.

While muscle fibers are in motion, there is an increase of blood flow, so they get nutrients and oxygen, digest them more fully than at a standstill period, and, as a result, products of metabolism are excreted more rapidly. During physical activity, there are changes in the composition of the muscles: they accumulate required substances (proteins, glycogen, enzymes) for their active work. Moreover, a better utilization of oxygen accelerates recovery processes [2].

Moderate physical activity reduces blood pressure, reduces heart rate and thus reduces the load on the heart and blood vessels. If we mention that it reduces cholesterol and thins the blood, then the value of the optimum motor activity in the prevention and treatment of cardiovascular diseases is very great (according to prevalence and the number of deaths, these diseases take the first place in the developed countries, including Russia) [2].There are dozens of diseases affecting a cardiovascular system. Physical activity has no positive effect only for some of them - a congenital heart disease, infectious diseases, allergic and inflammatory disease of the heart muscle and other membranes of the heart [1].

Physical activity prevents sclerosis of the large vessels, including the aorta. Pulse wave velocity among people who are regularly engaged in physical exercises, even in old age is much less than that of young people who do not deal with physical activity. This indicator is closely linked with the development of atherosclerosis [7].

Notable positive changes take place in the organs of external respiration: increasing the strength of the respiratory muscles, the size and mobility of the chest, as well as the vital capacity of lungs, which reflects functional capabilities of a person to withstand the load. Breathing becomes rare, but deep.

Physical exercises have a beneficial effect on blood. It increases red blood cells (RBCs) and hemoglobin, which delivers oxygen to tissues. A growing number of white blood cells (leukocytes) plays a major role in protecting the body from harmful influences.

During exercise periods, due to release of hormones (endorphins) into the blood, each person becomes refreshed and cheerful. [9] Such a method of boosting the energy does not lead to depression and prostration, which is typical for people using nervous system stimulants (coffee, drugs, alcohol, etc.).

It was determined that the blood of a tired man after performing physical exercises acquires the properties of a biological stimulant that exerts a positive impact on the processes healing chronic diseases (skin and internal organs).

Systematic physical exercises eliminate nervous tension, anxiety and irritability. It is the most effective way to deal with depressions, headaches and insomnia

Physical activity also has a positive effect on the immune system that protects a person from various infections and other diseases. This effect is more vivid when playing outdoors, when the effect of physical exercises is combined with hardening $[8,9]$.

When you exercise in the open air, there is a good ventilation of lungs, which eliminates stagnation and helps to prevent respiratory diseases.

A well-chosen set of exercises is capable to enhance and maintain basic physical qualities at an optimum level throughout the life and in old age [2].

MYa. Vilenskiy, VI Ilinich noted that Nordic walking trainings have a complex effect on the human body in different directions. Of all the types of physical activity, it is the most simple and accessible one.

Regular practice of Nordic walking has a variety of beneficial effects on the body, it helps to improve cardio - vascular and respiratory systems, activates the metabolism, increases gas exchange and oxidative processes. Large muscles act as a "peripheral heart" when they are activated during walking, thus improving the outflow of blood from the lower limbs, abdomen, pelvis.

Walking has a stimulating effect on the function of the digestive glands, liver, and intestines. This occurs as a consequence of a natural foot massage. Like other cyclical exercises, walking causes a favorable reorganization of the nervous processes, improves the activity of analyzers and the emotional state, and normalizes sleep.

Combining walking training with a low-calorie diet is preferable for the reduction of excess body weight.

Various authors (E.V. Milner E.V., Yablonsky I.M., V.I. Ilyinich, MJ Vilna, R.E.Motylyanskaya, M.P. Sotnikova etc.), whose opinions on recommended volume, intensity and duration of training differ, propose many options of the Nordic walking program.

In accordance with the level of physical conditions, the minimum, optimum and maximum allowable load characteristics are determined.

\section{Materials and methods}

The objective of this paper is to determine the impact of the method of selective graduated exercises on the body of women at the age of $50-60$, who are engaged in improving physical education.

The object of research is the process of physical training, and the subject is the optimal dosage of loads for women at the age of 50 - 60 years, during Nordic walking trainings.

Hypothesis: It is assumed that the use of graduated aerobic exercises, widespread in health and fitness studios, will improve the physical and functional condition of women at the age of 50-60. 
Hypothesis: It is assumed that the use of metered loads of aerobic focus in health and fitness classes will improve physical and functional condition of women at the age of 50-60.

The analysis of scientific - methodical literature has shown that there are many different methods of physical training, but the most popular and accessible view of improving physical training is Nordic walking, especially for people of mature and elderly age. Nordic walking is a kind of walking when you increase the intensity of exercises using hands, i.e. making hands heavier using special sticks.

Nordic walking began to develop in Europe in the 80s of the last century. Nordic walking is particularly popular in Scandinavian countries, particularly in Finland. Thus, the name of this kind of sport - the northern or Nordic walking - originated. For Russia, Nordic walking is a relatively new but rapidly growing form of physical activity. In the early 2000 s, Nordic walking has been presented in the most modern rehabilitation clinics, spa studios and fitness centers. In 2010, under the direction of Anastasia Poletaeva, first Russian professional "School of Nordic Walking" was opened. "Russian Club of Nordic Walking" was opened in Tomsk in 2013. According to some authors (Poletaeva A., etc.), Nordic walking has several advantages: it reduces the reserves of adipose tissue, increases the amount of lungs to $30 \%$, strengthens the heart muscle and increases the size of the heart, reduces blood pressure, reduces the heart rate, reduces the possibility of formation of blood clots. It increases and strengthens the muscles of the whole body, regulates the activity of the intestine, improves the sleep quality. Besides, there is a strong increase in hormones of happiness - endorphins and others.

One of the main advantages of Nordic walking is complete absence of contraindications and minimal risk of injury, which is very important for people of mature and elderly age.

\section{Results and discussion}

A group of women aged 50-60 years who expressed willingness to engage in Nordic walking was arranged to conduct the study. The pre-test was conducted to assess the level of physical readiness and the functional state.

The level of aerobic endurance for women was determined according to a 12-minute's Cooper test. According to the results of preliminary testing the women were divided into 3 groups.

Group 1 - a low level of physical readiness (from $850 \mathrm{~m}$ to $1100 \mathrm{~m}), 7$ persons.

Group 2 - an average level of physical readiness (from $1150 \mathrm{~m}$ to $1300 \mathrm{~m}), 4$ persons.

Group 3 - a high level of physical readiness (from $1350 \mathrm{~m}$ to $1550 \mathrm{~m}), 4$ persons.

The level of the maximum heart rate during training was assessed. [4, 7]. According to the recommendations of the World Health Organization (WHO), blood pressure was determined according to the formula:

Systolic blood pressure $=0,4 \mathrm{x}$ age +109

Diastolic blood pressure $=0,3 \times$ age +67
We determined the body mass index for women ( $\mathrm{P}$. Brock) $\mathrm{M}=\mathrm{H}-100$, where $\mathrm{M}$ - mass in $\mathrm{kg} ; \mathrm{H}$ - height in $\mathrm{cm}$.

Almost all women had high blood pressure, 12 of the 15 of women had disorders of fat metabolism.

Using testing (a SF-36 questionnaire), we determined the total well-being and satisfaction along with the percentage of those aspects of life that are affected by their state of health.

The SF-36 questionnaire was conducted among women engaged in intellectual work to determine their physical and mental health component as well as motivation for physical training.

All women who wished to participate in the experiment noted that they experienced rapid fatigue during the day, irritability, constant tiredness, affected sleep and high blood pressure, shortness of breath when walking upstairs for a few spans, excess weight and other complaints.

SF-36 of the physical component on the part of health showed that, as a whole, $53 \%$ of women estimated their health status as mediocre, $27 \%$ rated their health as good and $20 \%$ - as excellent. Most of the women reported that their health condition was at the same level as it had been a year ago. $60 \%$ of women said that their health was largely limited to heavy exercises (running, power sports, lifting and carrying heavy bags etc.). Over the past 4 weeks, $67 \%$ of women had to reduce the amount of time spent at work and other matters due to their physical condition. In addition, because of their physical condition $60 \%$ of women performed smaller volume of the planned work than they had scheduled and experienced difficulties in its implementation. $47 \%$ of women did not realise the level of their health condition, and $20 \%$ believed that their health condition was no worse than that of their friends. $13 \%$ said their health was excellent. $80 \%$ of women over the last 4 weeks experienced physical pain that prevented them from engaging in housework activity at home and outside the home.

The survey results obtained from the psychological component of health showed that the women's emotional state causes difficulties at work, and $53 \%$ of them performed less work than they had planned. $40 \%$ of the work performed is not as neat as usual. $47 \%$ of women said that their physical and emotional state interfered with their active communication with their families, friends and staff.

Despite their state of health, all the women expressed a desire to be engaged in Nordic walking. The main motives for engaging in the Nordic walking training are the following:

1. problems of the musculoskeletal system - 30\%;

2. excess weight $-30 \%$;

3. high (low) blood pressure $-80 \%$;

4. urban environment - $50 \%$;

5. lack of communication $-20 \%$;

6. enhanced fatigue $-60 \%$;

7. increased nervousness $-10 \%$;

8. dyspnea- $40 \%$;

9. sedentary lifestyle - necessity to move $-50 \%$;

10. prevention of diseases etc. 
To determine the optimal dose of physical activity women were proposed to pass a certain distance in 12 minutes at the pulse of $120-140$ beats per minute (Cooper test). During this time, every woman passed a certain distance, as a result all the women were divided into 3 groups according to their level of physical fitness. The main criteria for determining the optimal dose of load are objective and subjective fatigue indicators. [8] A special training plan was developed for each group during winter and snowless seasons, which specifies the optimum load parameters (volume, intensity).

Nordic walking training for women from the first, second and third groups during winter was held 3 times a week. HR during training for the first and second groups did not exceed 120-130 beats per minute, and for the third group it was 130-140 beats per minute.

Nordic walking training for women from 1,2 and 3 groups during winter is held 3 times a week. HR during training for 1 and 2 groups should not exceed 120-130 beats per minute, and for group 3 it should be 130-140 beats per minute.

The first group of women had to walk $2-3 \mathrm{~km}$ in $37-40$ minutes per one training. In the snowless period, the distance was $3-4 \mathrm{~km}$ in 33 - 45 minutes. For women from the second group in one class period it was necessary to walk 3-4 $\mathrm{km}$ in 35-50 minutes. In the snowfree period, the distance amounted to $4-5 \mathrm{~km}$ and had to be covered in $44-55$ minutes. The women from the third group had to walk $4-5 \mathrm{~km}$ in $45-60$ minutes. In the snowless period, $5-6 \mathrm{~km}$ had to be covered in $55-67$ minutes, so that the heart rate was equal to $130-140$ beats per minute, and individual trainings amounted to four times per week.

The analysis of the results of the experiment allowed us to estimate the main components of the psychological and physical health of women, as well as to find out the cause of decline in living standards and to set the basic motivation for physical training. The most significant factors that have a negative influence on the physical quality of life are age and a sedentary lifestyle. By the end of the experiment, $100 \%$ of the women estimated their general state of health and physical functioning as excellent, compared to their baseline indicators. The women, engaged in the arranged trainings, noticed an increase in their efficiency; their mood was lightened; they experienced greater cheerfulness; their sleep became sound; the blood pressure was normalized, and they lost $3-5 \mathrm{~kg}$ of their weight. There was a significant improvement of the results of the physical fitness among the women after the training. Besides, the women have improved the results in covering the distance of 500 $600 \mathrm{~m}$.

\section{Conclusion}

Thus, Nordic walking trainings, using individual graduated exercises and taking into account the peculiarities of women at the age of 50-60, have a positive impact on their overall health and psychoemotional state.

\section{Acknowledgment}

The results of the study were tested and put into practice for working with 50 - 60 year-old women, engaged in Nordic walking in sports and recreation groups and "Russian Club Nordic Walking" in Tomsk.

\section{References}

1. AA. Soboleva, RR. Khasanova, VI. Andreev, TV $>$ Sarycheva, LI. Smagly Abstract Book "The $8^{\text {th }}$ World Conference on Educational Science”, 325 (2016)

2. MYa. Vilenskiy, VI Ilinich Physical training of intellectual workers. St. Petersburg: Drofa (1997)

3. A. Poletaeva, Nordic Walking. Health with a light step. St. Petersburg: Piter (2013)

4. P. Kocur, M. Wiernicka, . M. Wilski, E. Kaminska, L. Furmaniuk, M. Flis Maslowska, J. Lewandowski, J. Phys. Ther. Sci., 27 (2015)

5. J. Perry, JM. Burnfield, SLACK Incorporated (2010)

6. T. Parkatti, J. Perttunen, P. Wacker, J. Aging Phys Act, 20 (2012)

7. M. Tschentscher, D. Niederseer, J. Niebauer, Am J Prev Med, 44 (2013)

8. N. Takeshima, MM. Islam, ME. Rogers et al, J Sports Sci Med, 12 (2013)

9. SD. Park, SH. Yu, J. Of Physical Therapy Science, 27 (2015) 